\title{
TRANSICIÓN A UNA CIENCIA Y CULTURA TRANSDISCIPLINARIAS
}

\author{
James Morin St. Onge ${ }^{1}$
}

\section{Resumen/Abstract}

La transdisciplinariedad busca el desarrollo de conocimientos científicos, significados culturales y políticas sociales que respondan a los complejos problemas de nuestros tiempos. Revisaremos primero, en perspectiva histórica, algunos criterios, métodos y teorías que han orientado el desarrollo de esta propuesta transformativa. Luego nos centramos en el problema epistemológico sobre cómo integrar la diversidad de saberes involucrados en la resolución de problemas complejos. Frente a este desafío, proponemos un método que orienta la apropiación de la estructura dinámica de la propia conciencia intencional, como base común a todas las formas auténticas del saber. Sobre esta base presentamos un modelo para orientar el diálogo y la colaboración transdisciplinaria entre el sentido común, las expresiones artísticas, las teorías científicas, los significados culturales, las convicciones religiosas, los derechos políticos y el razonamiento filosófico.

Palabras clave: transdisciplinariedad, conciencia intencional, método empírico general.

\section{TRANSITION TO A SCIENCE AND TRANSDISCIPLINARY CULTURE}

Transdisciplinary promotes developing scientific knowledge, cultural meanings and social policies that respond to the complex problems of our times. First, we review, in historical perspective, criteria, methods and theories that have oriented the development of this transformative proposal. Then we focus on the epistemological problem on how to integrate the diversity of knowledge involved in the solution of complex problems. To face this challenge we propose a method that promotes appropriating the dynamic structure of one's intentional consciousness, which is common to all forms of authentic knowledge. On this basis, we present a model for guiding dialogue and transdisciplinary collaboration between common sense, artistic expressions, scientific theories, cultural meanings, religious convictions, political rights and philosophical reasoning.

Keywords: transdisciplinary, conscious intentionality, generalized empirical method.

\footnotetext{
${ }^{1}$ Canadiense con permanencia definitiva en Chile. Universidad Católica del Maule. E-mail: jmorinso@gmail.com
} 


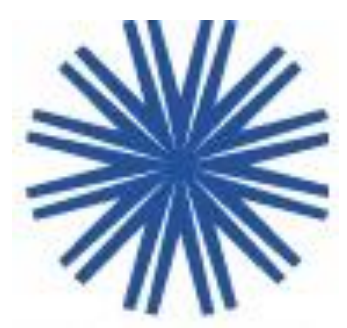

Conocimiento transdisciplinario: historia, desafios, preguntas y criterios

Se puede caracterizar el enfoque transdisciplinario como un cambio de paradigma en la producción del conocimiento. Busca superar la división del trabajo académico en disciplinas administradas y segmentadas en departamentos separados entre sí. Como alternativa, propone convocar a investigadores y actores sociales no académicos en la producción e integración de saberes transformativos que responden a los complejos problemas del mundo actual (Hirsch, 2008, p.29). A continuación, revisamos los orígenes de la propuesta transdisciplinaria y su desarrollo histórico. Buscamos identificar algunos pensadores, conceptos, criterios, enfoques metodológicos y supuestos teóricos que han orientado el desarrollo de esta propuesta.

La primera definición ampliamente conocida de la transdisciplinariedad surgió en un seminario sobre la interdisciplinariedad en la enseñanza e investigación universitaria, que se realizó en la Universidad de Niza en 1970 con el patrocinio del Ministerio de Educación francés y de la Organización para la Cooperación Económica y el Desarrollo. (Lawrence, 2010). En este contexto el psicólogo cognitivo Jean Piaget, el astrofísico Erich Jantsch y el físico matemático Andre Lichnerowicz propusieron un enfoque transdisciplinario capaz de moverse entre, a través y más allá de los límites de diferentes disciplinas, con el fin de buscar una comprensión integral y sistémica de los niveles de la realidad que intervienen en problemas complejos. Piaget (1972), conocido por su teoría del desarrollo cognitivo y epistemología genética, caracterizó la transdisciplinariedad como una etapa superior en el desarrollo de las estructuras del pensamiento, que conduce a una ciencia general capaz de lograr una asimilación recíproca entre disciplinas. Jantsch (1972) propuso fundamentar la transdisciplinariedad sobre axiomática generales y derivadas de la articulación entre epistemologías que buscan coordinarse sistémicamente en torno a un propósito global. Lichnerowicz (1972) planteó la matemática como lenguaje común para la identificación de estructuras elementales entre las ciencias y la tecnología. El mismo año, una tesis doctoral 
sobre transdisciplinariedad y ciencias humanas, examina la literatura de la época sobre filosofía y las ciencias sociales (Mahan, 1972). La investigación constata que ya existían críticas a la segmentación de disciplinas, y que había propuestas novedosas que sintetizaban conocimientos con consideraciones éticas para mejorar la sociedad. Pasaron casi dos décadas antes que se dieron las condiciones para retomar nuevamente el tema.

En 1987 se formó el Centro Internacional de Investigaciones y Estudios Transdisciplinarios (CIRET). Auspiciado por la UNESCO, en 1994 el Centro organizó, en Portugal, el Primer Congreso Mundial de Transdisciplinariedad, contexto en que sus participantes firmaron una declaración de criterios y recomendaciones para orientar el desarrollo de la transdisciplinariedad (Camus, 1997). En el Congreso y su declaración, se nota las contribuciones de Basarab Nicolescu, físico teórico rumano y director del Centro, y del filósofo y sociólogo francés Edgar Morin.

Nicolescu (2009), postula tres principios básicos de la transdisciplinariedad, por los cuales se: 1) reconoce la existencia de diferentes niveles de la realidad, regidos por lógicas diferentes; 2) acepta una lógica del tercero incluido en que elementos contradictorios y mutuamente excluyentes, pueden complementarse en otro nivel de realidad; y 3) afirma que la complejidad que está en todos partes exige un enfoque más amplio. Morin contribuyó con una mirada desde el pensamiento complejo, que pone énfasis sobre lo emergente, lo imprevisible, lo auto-organizativo y lo aleatorio, posición que contrasta con las certezas de las leyes invariables del saber científico tradicional. Como forma de acercarse a una realidad compleja, Morin (2001) acentúan tres operaciones. Estas incluyen: 1) distinguir entre diferentes campos de saber, con sus objetos de estudio, estructuras teóricas y metodológicas propias; 2) conjugar para promover el diálogo y potenciar la asociación entre diferentes formas de saber sin reduccionismos; y 3 ) implicar distintos campos de conocimiento y actores sociales, en la búsqueda de propuestas a nivel sistémico, con una actitud abierta a lo desconocido (Carrizo, 2003).

El mismo año que tuvo lugar el Primer Congreso, se publicó un libro que ofrece un acercamiento menos filosófico y más pragmático al desarrollo de la transdisciplinariedad. 
$\overline{\text { Michael Gibbons (1994) y sus colegas contaban con experiencias en la realización de }}$ proyectos colaborativos entre las ciencias sociales, la medicina, la arquitectura, la tecnología, la economía, las humanidades, la informática y la educación. Estos proyectos respondían a problema específicos e incidían sobre el desarrollo de políticas públicas. Concibieron como Modo 2, la forma de producir conocimientos que involucra a expertos provenientes de la academia, el gobierno, la industria y organizaciones no gubernamentales en el desarrollo de propuestas para la resolución de problemas específicos. Esta mirada más descriptiva, analítica y práctica amplió la comprensión de la transdisciplinariedad desde la sociología de las ciencias, que considera las funciones de la tecnología, la educación y el diseño de políticas en la producción del conocimiento (Bernstein, 2015).

En 1998 la UNESCO organizó un simposio para aclarar la función sinergética de la transdisciplinariedad en la integración del conocimiento. El informe del simposio define la multidisciplinariedad como yuxtaposición de distintas contribuciones disciplinarias que no tiende a producir perspectivas unificadas. La interdisciplinariedad, busca reunir puntos de vistas distintos, pero no logra una mirada coherente en la medida que no supera la perspectiva de disciplinas fragmentadas. La transdisciplinariedad, en cambio, busca un marco común que pone atención a la interacción entre diversos campos del saber y busca un meta-lenguaje para la integración del conocimiento entre diversos niveles de la realidad. (UNESCO, p.18, 1998).

En el simposio Anthony McMichael (1998) señaló que los métodos mono-disciplinares tradicionales limitan la mirada de las ciencias, al reducir realidades complejas a unos componentes cuantificable separados de su totalidad. En cambio, la transdisciplinariedad amplía el horizonte cuando explora nuevas dimensiones de incertidumbre, sin temer la ignorancia inicial que anima la búsqueda del conocimiento. McMichael afirmó que la transdisciplinariedad va más allá de la interdisciplinaridad y la multidisciplinariedad. Refiere a algo más complejo que la yuxtaposición o combinación de diferentes campos disciplinarios. En esta perspectiva el todo no es sólo mayor que la suma de sus partes disciplinarias, sino que tiene propiedades emergentes cualitativamente diferentes. Agregó que la transdisciplinariedad requiere la cooperación, para poder lograr la sinergia que se produce al 
buscar en común respuestas a las preguntas de investigación formuladas por diversas ciencias y actores sociales, respecto a problemas complejos y sistemas dinámicos.

Desde el principio de este siglo varias fundaciones, instituciones de educación superior y gobiernos realizaron inversiones significativas para fomentar el desarrollo de centros y equipos transdisciplinarios. Esto incentivó la organización de grupos de lectura y conferencias, el desarrollo de programas de estudio y la entrega de premios de reconocimiento a actores y organizaciones destacados que contribuyen a la colaboración transdisciplinaria. Se consolidaron programas de investigación, propuestas curriculares y estrategias pedagógicas que promueven el desarrollo de habilidades transdisciplinarias. El desarrollo de tesis doctorales, los resultados de aplicaciones prácticas, las actas de conferencias internacionales, bibliografías especializadas y diversas publicaciones científicas dedicadas al tema contribuyeron a consolidar la transdisciplinariedad como campo de conocimiento. De 1970 y 1985, aparecían entre cero a cinco publicaciones por año, con un total de 30 a lo largo de 15 años. En 2005 el número había subido a setenta y cinco publicaciones al año. (Kueffer, 2007).

El Centro Internacional de Investigaciones y Estudios Transdisciplinarios, ${ }^{2}$ fundado en 1987, estudia el flujo de información entre las distintas ramas del conocimiento, con el fin de comprender la unidad en medio de la diversidad y favorecer el diálogo entre diferentes ámbitos del conocimiento. El Centro ofrece diversos recursos que incluyen su Proyecto Moral, la Carta de la Transdisciplinariedad, una colección de investigaciones, libros y tesis sobre el tema. Otra agrupación, la Academy of Transdisciplinary Learning and Advanced Studies (ATLAS), fundada en el 2000, promueve la colaboración en torno a investigaciones y proyectos transdisciplinarios que favorecen al desarrollo sostenible social, ambiental, económico y ético en todo el mundo. ${ }^{3}$ Ofrece recursos en línea que incluyen módulos, libros e informes.

2 Sitio web de la International Center for Transdisciplinary Research (CIRET). En http://cirettransdisciplinarity.org/index_en.php.

3 Sitio web de la Academy of Transdisciplinary Learning and Advanced Studies (ATLAS). En http://www.theatlas.org/index.php/about-atlas. 
La Network for Transdisciplinary Research (td-net) ${ }^{4}$ es una iniciativa de las Academias Suizas de Artes y Ciencias. Fue inaugurada en un congreso de Zurich en 2000, con representantes de 51 países. En esta agrupación las disciplinas no son el foco de la investigación, sino las preguntas y las prácticas que buscan la resolución de problemas complejos. Realizan estudios en los campos de la interacción humana con sistemas naturales (agricultura, silvicultura, industria, mega ciudades) y de desarrollo técnico (nuclear y biotecnología, genética). La td-net no propone una orientación metodológica universal. Favorece más bien una elección informada a partir de un repertorio de métodos, instrumentos y estrategias que incluyen el desarrollo de hipótesis, la construcción de modelos y procedimientos de evaluación integradora (Bergmann, 2012). Propone un enfoque heurístico que articula la transdisciplinariedad en torno a la búsqueda de respuestas a las preguntas de investigadores, quienes rinden cuenta de sus contribuciones para resolver problemas complejos (Huutoniemi, 2014).

Otra iniciativa, respaldada por el Instituto Nacional del Cáncer, abarca el campo denominado como la ciencia de la ciencia en equipo (SciTS). Investiga las condiciones, los procesos de colaboración y los resultados asociados con iniciativas científicas desarrollados por equipos. Estudia los descubrimientos, resultados educativos y las traducciones de los hallazgos en nuevas prácticas, patentes, productos, avances técnicos y políticas. Brinda juegos de herramientas, recursos bibliográficos y noticias sobre eventos, financiamiento y ofertas de empleo.

\section{Características y criterios transdisciplinarios}

El desarrollo sostenido de una multiplicidad de proyectos transdisciplinarios ha permitido categorizar e investigar esta propuesta en torno a: campos de aplicación, disciplinas involucradas, enfoques metodológicos, perspectivas meta-teóricas, contextos de aplicación y herramientas usadas. El estudio de la estructura del trabajo en el diseño, la gestión y la evaluación de investigaciones y prácticas transdisciplinarias (Krott, 2003) ha servido a su vez

\footnotetext{
${ }^{4}$ Sitio web de la Network of Transdisciplinary Research. En http://www.transdisciplinarity.ch/en/td-net/Uebertd-net.html.
} 
para orientar la definición de políticas públicas (Kastenhofer, 2009) en el desarrollo de este campo novedoso.

Se ha situado la transdisciplinariedad dentro una cuarta etapa en relación con la evolución moderna de las ciencias y la tecnología. 1) La primera respondió a la necesidad de desarrollar disciplinas autónomas con métodos y técnicas propias. La jerga especializada que desarrollaron facilitó una colaboración intra-disciplinaria efectiva, pero dificultó la comunicación interdisciplinaria y endureció los muros entre disciplinas. 2) Una segunda etapa de colaboración entre las ciencias y la tecnología aceleró la producción industrial y la expansión del comercio global. 3) En una tercera etapa, con la informática se alcanzó el manejo de mayores niveles de complejidad mediante enfoques sistémicos e integrados, que para su funcionamiento requerían equipos de diseño y de colaboración entre universidades y el sector industrial. 4) En una cuarta etapa, más reciente, los problemas mundiales relacionados con la salud, el medio ambiente y los conflictos que amenazan a la paz, requieren enfoques de mediación que van más allá de la colaboración entre disciplinas. ${ }^{5}$ Además de la colaboración de las ciencias básicas y sociales, se exige el manejo de competencias y conocimientos que transcienden los límites de las ciencias, la tecnología y la producción. Requiere una visión filosófica y ética sobre la interdependencia de la gente, combinada con la capacidad de dialogar con diferentes idiomas, valores culturales, tradiciones étnicas, visiones políticas y creencias religiosas, que juntos inciden para formar percepciones y orientar la participación ciudadana en la resolución de los problemas.

Centenares de proyectos transdisciplinarios han respondido a una diversidad de situaciones complejas, que han sido caracterizadas como problemas perversos (Brown, 2010). Estos tienen relación con el cambio climático, las epidemias de salud, los recursos renovables y no renovables, el terrorismo, y la planificación urbana. Ejemplos incluyen: datos de salud sobre individuos, poblaciones y ecosistemas (Conrad, 2009); tratamiento sistémico de problemas crónicos, cómo la violencia doméstica, el abuso de sustancias, personas sin hogar y refugiados (Kirst, 2011); la relación entre ingeniería y medio ambiente (Godemann, 2008);

\footnotetext{
${ }^{5}$ The ATLAS. Transdisciplinarity. En http://www.theatlas.org/index.php/about-atlas-2.
} 
servicios eco-sistémicos en la gestión de la tierra y el agua (Siew, 2012); ciencia climática en la toma de decisiones políticas (Newsom, 2016); comunicación en afectividad y sexualidad entre familia y escuela a lo largo del ciclo vital (Morin, 1996); estudios de movimientos sociales que favorecen la justicia en relación con la pobreza, la inequidad, la injusticia y la discriminación por razón de género, raza, etnia, religión o sexualidad (Asumah, 2015); tecnología, sociedad y religión en perspectiva de las humanidades ambientales (Deane-Drummond, 2015); bases para una economía ecológica (Spash, 2012). Manfred MaxNeef (2005) ha identificado cinco problemas, que a largo plazo tendrán un impacto global y requerían respuestas transdisciplinarias: el uso del agua, las migraciones forzosas, la pobreza, la crisis ambiental, la violencia y el terrorismo, el neo-imperialismo y la destrucción de los tejidos sociales.

En el conocimiento transdisciplinario Max-Neff (2005) distingue cuatro niveles, cada uno definido por la búsqueda heurística de respuestas a una pregunta clave en torno a la cual se reúnen las disciplinas correspondientes. 1) El nivel empírico pregunta sobre lo que existe y busca respuestas en la matemática, la física, la química, la biología, la psicología, la sociología y la economía. 2) El nivel pragmático pregunta sobre lo que somos capaces de hacer y busca respuestas en disciplinas tecnológicas que ofrecen orientaciones propositivas como la arquitectura, la ingeniería, la agronomía, la industria y el comercio. 3) El nivel normativo pregunta sobre qué queremos hacer y busca orientaciones formales en la planificación, el diseño, las políticas y la ley. 4) El cuarto nivel moral pregunta sobre qué debemos hacer y busca criterios epistemológicos y valóricos en la ética y la filosofía.

En la producción de conocimientos transdisciplinarios la convergencia y la integración son claves. La convergencia busca articular datos, métodos, teorías y modelos que facilitan la comprensión entre diversos campos de saber. Por el carácter complejo de los problemas transdisciplinarios, se requiere niveles de convergencia académica y social. A nivel académico se busca articular las ciencias naturales y sociales con las humanidades, las matemáticas, la ingeniería y la informática. A nivel social se busca una colaboración sistémica para desarrollar estrategias estructurales a nivel social, cultural y política, para 
promover aprendizajes y cambios conductuales que aseguran soluciones comunitarias que perduren en el tiempo (National Research Council, 2014).

Dado que el trabajo en equipo es clave para la integración, se ha estudiado cómo los equipos gestionan información y conocimientos que orientan las prácticas de colaboración transdisciplinaria (Bergmann, 2012). Investigaciones consideran los modelos organizacionales para la articulación de sistemas humanos y medioambientales complejos. Estudian los marcos teóricos para aclarar supuestos ontológicos, enfoques epistemológicos, procedimientos metodológicos y normas organizacionales, y la retro-alimentación y adaptación de estos (Scholz, 2006). Los proyectos transdisciplinarios se convocan en torno al problema que se propone responder. Su gestión abarca la formulación de preguntas e hipótesis, la definición de metodologías, el diseño e implementación de estrategias, y el análisis y evaluación de resultados. Existen matrices para evaluar la división del trabajo y los logros de integración en relación con metas, bienes y valores comunes (Burger, 2003). Consideran la colaboración en equipo, la confianza y respeto entre participantes, y el impacto de la propuesta (Wiesmann, 2008).

La sostenibilidad global, por ejemplo, requiere una colaboración científica y social para la integración del conocimiento transdisciplinario en el desarrollo de programas efectivos. En este proceso se distinguen tres dimensiones de integración científica, internacional y sectorial; desarrollada en tres etapas de colaboración en el codiseño de investigaciones, en la coproducción de conocimientos y en la codifusión de los resultados (Mauser, 2003). A nivel comunicacional, se han diferenciado niveles semánticos ordinario, técnico, formal y metateórico para facilitar el entendimiento y la colaboración entre los actores de diversos sistemas (Hinkel, 2008). Se distinguen también entre niveles conceptuales altos y bajos en la integración del conocimiento entre diversas disciplinas y sectores sociales. Los conceptos altos comprenden los siguientes aspectos esenciales de cualquier problema significativo: dinámica y sistema, organización y escala, modelos de control, gestión y política, adaptación y aprendizaje e historia. Los conceptos de bajo nivel son derivados de las disciplinas y sirven de nexos entre estas (Newell, 2005). 


\section{Enfoques metodológicos y fundamentos teóricos}

Investigaciones sobre las prácticas transdisciplinarias han permitido distinguir métodos y bases teóricos que orientan la colaboración e la integración transdisciplinaria del conocimiento. Se ha identificado 43 métodos de integración transdisciplinaria, que han sido agrupados en siete clases. 1) La clarificación conceptual y enmarcamiento teórico para facilitar la integración cognitivo y social. 2) La formulación de preguntas e hipótesis en torno a problemas para articular la investigación científica y la praxis de los actores sociales. 3) El diseño de nuevos métodos entre disciplinas, orientados al problema para la integración de conocimientos eficaces. 4) La evaluación multicriterio que consideran las expectativas y las contribuciones de los científicos y actores sociales involucrados. 5) El desarrollo de modelos que faciliten la integración de información entre diferentes campos científicos y de acción social. 6) El desarrollo de servicios, publicaciones y productos para los campos científico, político y de negocios. 7) La definición de procedimientos cooperativos para formar equipos y bases institucionales que aseguren la integración cognitiva y social de conocimientos transdisciplinarios que perduran en el tiempo (Godemann, 2008).

Otra investigación ha diferenciado cinco enfoques transdisciplinarios. Incluyen: 1) lenguaje figurativo que relaciona las disciplinas con un problema; 2) experiencias personales que buscan respuestas a problemas del entorno; 3) meta-teorías y lógicas formales para integrar conocimientos de diversas disciplinas; 4) Metáforas profundas y generativas que tienen implicaciones cognitivas integrativas; y 5) una combinación de todas las formas anteriores (Judge, 2005). El autor afirma además que el humor y el juego pueden facilitar a la integración de conocimientos transdisciplinarios (Judge, 2010).

Se distingue tres discursos que buscan orientar el desarrollo transdisciplinario. Estos incluyen: 1) la búsqueda de una trascendencia epistemológica; 2) la resolución de problemas complejos: y 3) la transgresión de límites disciplinarios. El discurso de la transcendencia busca la unidad del conocimiento para responder al problema de la fragmentación del saber en disciplinas especializadas. En este discurso se distingue dos opiniones sobre si se debe 
buscar la unidad a través de una teoría universal o por una articulación de la unidad entre cosmovisiones diversas. El discurso sobre la resolución de problemas busca desarrollar la transdisciplinariedad en torno a fines pragmáticos y las necesidades de la comunidad, como el desarrollo social, ambiental y económico sostenible. Esto exige que las universidades realicen su misión social por medio de innovaciones científicas y educativas que aseguran una retroalimentación continua sobre la interacción entre sistemas naturales, sociales, técnicos, valóricos y de significación cultural. El discurso de la trasgresión cuestiona las lógicas instrumentales, las suposiciones reduccionistas y la dicotomía entre expertos en el desarrollo de un conocimiento dominante que margina otras formas de saber. Fomenta nuevas asociaciones para la producción contextualizada de conocimientos socialmente responsables que plantean cuestiones de justicia sociopolítica sobre temas relacionados con identidades indígenas, discriminación por razón de clase, género, raza o etnia, y temas relacionados con el desarrollo sostenible (Klein, 2014).

Ahora bien, la diversidad de problemas complejos que la transdisciplinariedad confronta, y la variedad de enfoques y prácticas que ha inspirado para el desarrollo de conocimientos transformativos, hace preguntar sobre el estatus epistemológico de esta propuesta. Proponemos abordar este problema por medio de la pregunta siguiente. ¿Cómo y con qué bases se puede producir conocimientos válidos sobre problemas complejos en que están involucrados diferentes marcos teóricos, múltiples enfoques metodológicos, distintas organizaciones, diversos grupos de interés, y escalas de valores culturales heterogéneos?

Ahora bien, buscar la integración del conocimiento no es patrimonio exclusivo de la transdisciplinariedad. Aparece como anhelo en la filosofía naciente de la antigua Grecia, en la Summa teológica medieval, así como en la búsqueda ilustrada de promover el progreso por medio de una razón universal. Durante los Siglo XIX y XX se buscó la unidad del conocimiento por medio de la fenomenología, el marxismo, el estructuralismo, enfoques sistémicos, la cibernética, el feminismo y la sociobiología. Estas propuestas ofrecen enfoques comprensivos para la integración, que por otro lado pueden volverse problemáticos si reducen los fenómenos a una ideología o si tales enfoques se convierten en proyectos monolíticos o sistemas cerrados (Klein, 1998). 
En la búsqueda de la integración del conocimiento se tiende a distinguir entre dos direcciones u opciones aparentemente excluyentes una de la otra. En una de estas opciones se supone que existe una unidad en el mundo que puede ser percibida. Esta unidad está concebida como un orden del ser, una unidad ontológica, constituida por su naturaleza común y conocida por las ciencias. La otra dirección se basa en el supuesto de que la unidad es una construcción del sujeto humano, basada en sus operaciones cognitivas. En esta perspectiva, la realidad no sería algo que pudiera ser descubierto como un orden objetivo existente, sino mediante la comprensión subjetiva (Hirsch, 2002).

Esto levanta otra pregunta crítica. ¿Será posible una integración de las dimensiones subjetiva y objetiva del conocimiento? Basarab Nicolescu postula una lógica del tercero incluido en que elementos aparentemente contradictorios y mutuamente excluyentes, pueden complementarse en otro nivel de realidad. Él afirma que la física cuántica -con sus nociones de superposición de estados, no separabilidad, causalidad global e indeterminismo- replantea el problema de la separación objeto-sujeto. Cita a Werner Heisenberg, Premio Nobel de Física, quien propuso: superar la rígida división entre una realidad objetiva y una subjetiva; renunciar a la referencia privilegiada a la exterioridad del mundo material; y situar la verdadera filosofía en el umbral entre la ciencia y la poesía. Nicolescu explica que la noción de "más allá de las disciplinas" significa precisamente la interacción sujeto-objeto y que la trascendencia inherente a la transdisciplinariedad afirma el sujeto que conoce. Esto implica reconocer que toda ciencia es producida por la conciencia de seres humanos que conocen, sujetos que, en las ciencias tradicionales, tienden a desaparecer en las formas de conocer delimitadas por disciplinas que solo atienden al objeto de conocimiento (Nicolescu, 2010). El biólogo chileno Humberto Maturana coincide al señalar que la humanidad vive en un estado enajenado por la división del ser en lo material y en lo espiritual, lo que impide ver que lo espiritual es un estado de conciencia y modo de vivir la propia corporalidad (Maturana, 1997).

Sin embargo, el cuestionamiento más crítico tiene relación con la propuesta de fundamentar la transdisciplinariedad en relación con la teoría de la complejidad o del caos. A nivel 
epistemológico, este enfoque no define un cuerpo integrado de conocimiento. Refiere más bien a una variedad de teorías relacionadas con la no-linealidad, la cibernética, los objetos fractales, la nueva termodinámica, la autopoiesis, la teoría de sistemas, los autómatas autoorganizados, el principio de generación de orden a partir del ruido, la teoría del azar organizador. Aun cuando este enfoque abre nuevos horizontes de comprensión sobre los diversos niveles de la realidad, la diversidad teórica incluida en el pensamiento complejo levantada dudas sobre la viabilidad epistemológica de articular la transdisciplinariedad en torno a este enfoque teórico. También se cuestiona la validez de aplicar en el ámbito social y cultural modelos desarrollados por la física, la cibernética y la biología. Carrizo recuerda por ejemplo la historia problemática de los intentos de aplicar a las ciencias sociales unos modelos basados en teorías mecánicas y evolucionistas (2004). Actualmente es ilustrativo el cuestionamiento crítico que los biólogos Humberto Maturana y Franscisco Varela han levantado sobre la propuesta del sociólogo Niklas Luhmann, quien aplicó la teoría biológica de la autopoiesis a los sistemas sociales (Rodríguez, 2003).

Con estas consideraciones, en la sección siguiente presentamos un enfoque que aporta criterios para orientar el diálogo y la colaboración entre diversos campos del saber. Este enfoque está basado sobre las operaciones conscientes e intencionales, de observar, entender, juzgar y decidir, actividad común y constitutiva del conocimiento en cualquier campo del saber humano. Al promover apropiar estas operaciones de la conciencia intencional propia, este enfoque fomenta el protagonismo de los sujetos y ofrece un marco común para el dialogo y la colaboración, sin imponer métodos, conceptos y normas ajenos a los distintos campos del conocimiento.

\section{Método de articulación transdisciplinaria}

Los fundamentos epistemológicos que proponemos para articular el diálogo y la colaboración transdisciplinaria están fundados sobre la experiencia de entender. En su obra Insight: un estudio sobre la comprensión humana, el filósofo canadiense Bernard Lonergan (1904 1984) invita al lector de observar, comprender, afirmar y apropiar su propia experiencia de entender. Con esta finalidad nos lleva a experimentar y diferenciar la estructura dinámica de 
la propia conciencia intencional que sustenta el desarrollo del conocimiento en todos los campos del saber humano.

Apropiación consciente e intencional de la actividad de conocer

Lonergan afirma que se puede comprender la experiencia de entender mediante las respuestas a tres preguntas. ¿Qué hago cuando conozco? ¿Por qué esto es conocer? ¿Qué conozco cuando hago esto? Su obra Insight está estructurada para responder a estas preguntas. ${ }^{6}$ A continuación, sintetizamos sus respuestas a estas preguntas. ${ }^{7}$

La respuesta a la primera pregunta (¿qué hago cuando conozco?) se encuentra al observar la propia actividad humana de entender. Es una actividad compuesta por las operaciones cognitivas de observar, inquirir, comprender, formular lo entendido, verificar con fundamentos y deliberar para decidir lo que corresponde hacer con libertad responsable. Juntas, estas operaciones constituyen cuatro niveles de consciencia intencional diferenciados, articulados e inseparables: y son el nivel de lo sensible, inteligible, racional y moral. Estas operaciones, que definen la estructura dinámica de la propia consciencia intencional, fundamentan un método empírico generalizado.

Es un método empírico porque describe las operaciones que constituyen la experiencia de conocer. Es un método generalizado porque este no se limita al dato de los sentidos, sino que incluye también al dato empírico de las operaciones intencionales de la consciencia inteligible, racional y responsable, que son integrales a, y constitutivas de la experiencia de conocer. Es un método trascendental en cuanto la propia consciencia intencional exige que uno sea atento, inteligible, racional y responsable para poder conocer con objetividad. Sobre este método Lonergan fundamenta su teoría del conocimiento. Afirma que en estas operaciones se encuentra el núcleo de las normas que son comunes a y sustentan el desarrollo diferenciado del sentido común, las teorías científicas, los significados culturales, las convicciones religiosas y el razonamiento filosófico (Lonergan, 1988, p.29).

\footnotetext{
6 Cf. Lonergan, Bernard. (1992), Insight. Editorial Notes f [16], p. 779.

${ }^{7}$ Los tres párrafos siguientes están basados en un resumen de la sección 4. "Las Funciones del Método Trascendental” del capítulo 1. Método. Cf. Lonergan, Bernard. (2006). Método en Teología. pp. 26-32.
} 
La respuesta a la segunda pregunta (¿por qué esto es conocer?) fundamenta una epistemología. Esta explica por qué se conoce, mediante las operaciones cognitivas de la consciencia intencional, en la medida en que se transciende respecto a los objetos o sujetos que se desea conocer. Este proceso está animado por el deseo de saber, lo que despierta la curiosidad y levanta preguntas que orientan la búsqueda de respuestas. En este proceso heurístico las percepciones, la imaginación y la memoria proveen imágenes en que la inteligencia puede captar en los datos la forma de lo inteligible. Las expresiones de jaja! o eureka, manifiestan la experiencia pre-conceptual del insight, del chispazo inteligible, del descubrimiento que posibilita el entendimiento, y la formulación posterior con conceptos, explicaciones o interpretaciones. La misma experiencia del entendimiento es lo que despierta las exigencias de la consciencia racional que requiere evidencia suficiente para poder fundamentar juicios sobre lo que es probable, real y de valor. Por esta razón esta posición epistemológica se define como realismo crítico, en cuanto no reduce el conocimiento al dato empírico o a ideas o conceptos. Su posición afirma que el conocimiento está fundado por un juicio que verifica cómo el entendimiento explica o interpreta adecuadamente el dato y que al mismo tiempo responde a las preguntas, dudas y criticas relevantes. Sobre esta misma base, la consciencia moral encuentra sus criterios para fundamentar su conocimiento concreto del bien humano.

La respuesta a la tercera pregunta (¿qué conozco cuando hago esto?) define una metafísica heurística y crítica que está basada en el deseo natural de conocer la realidad. La realidad, o la noción del ser, no tiene restricción. Incluye todo lo conocido y todo que queda por conocer y abarca tanto el ser proporcionado como el ser trascendental de la realidad. El conocimiento del ser proporcionado está mediado por el sentido común, las matemáticas y las ciencias naturales y humanas. El conocimiento trascendental de la belleza, de la verdad, del bien y de Dios esta mediado por el arte, la filosofía, la ética y la fe. Lonergan (1988) señala:

... la objetividad es sencillamente la consecuencia de la subjetividad auténtica, de la atención genuina, de la inteligencia genuina, de la racionabilidad genuina y de la responsabilidad genuina. Las matemáticas, las ciencias, la filosofía, la ética, la teología difieren de muchas maneras; pero tienen el rasgo común de que su objetividad es el fruto de la atención, inteligencia, racionabilidad y responsabilidad (p.29). 
La apropiación de la propia conciencia intencional en el acto de conocer aporta orientaciones prácticas y teóricas para orientar el diálogo y la colaboración transdisciplinaria entre diversas formas de saber. 1) Es un enfoque que promueve el protagonismo de sujetos conscientes e intencionales, quienes transcienden en relación con lo que desean conocer. 2) La actividad de la conciencia intencional de observar, entender, juzgar y decidir es común a y da unidad a una ciencia y a las ciencias. 3) La experiencia del insight, que capta la forma inteligible en los datos, es la condición de posibilidad para todo concepto, teoría y juicio que busca una síntesis unificadora del conocimiento. 4) La apropiación de la propia interioridad (consciencia intencional) permite distinguir, relacionar y unificar los distintos puntos de observación que las diversas formas de saber aporten sobre la misma realidad.

Modelo para orientar la colaboración transdisciplinaria

Lonergan propone que el método empírico generalizado, que está fundado sobre la estructura dinámica de la consciencia intencional, puede servir como un modelo, no para ser imitado o copiado, sino como un marco que facilita el diálogo y la colaboración para desarrollar una síntesis e integración superior del conocimiento. Señala que los modelos son para las ciencias sociales, las filosofías y las teologías, como las matemáticas lo son para las ciencias naturales. Los modelos no pretenden ser descripciones de la realidad, sino simplemente son grupos entrelazados e inteligibles de términos y relaciones que son útiles para orientar la atención de un investigador, guiar su estudio, facilitar la comunicación en la formulación de hipótesis y la interpretación de realidades complejas (Lonergan, 1988, pp.10 y 277-278).

Una característica del modelo es su estructura isomórfica y sistémica. Un isomorfismo nos remite a la similitud de formas entre entidades distintas y relacionadas. Por ejemplo, se relacionan y se integran las matemáticas y las ciencias empíricas mediante operaciones isomórficas que pueden ser concretas, numéricas y/o simbólicas (Lonergan, 1999, p.381). También el universo del ser es isomórfico con las operaciones básicas de experimentar, entender y juzgar (Lonergan, 1993, p.131), lo que permite examinar la relación isomórfica entre la actividad de la conciencia intencional, la estructura del conocimiento y la comunicación del saber (Lonergan, 1999, pp.475, 642-643). Así se puede distinguir entre las 
ciencias del sujeto (conciencia intencional), las ciencias de la objetivación (método empírico generalizado) y las ciencias del objeto (universo del ser). Es sobre la base de esta diferenciación articulada entre ciencias, que postulamos la posibilidad de fundamentar el diálogo y la colaboración transdisciplinaria entre los diversos campos de saber.

El modelo que presentamos a continuación (Tabla 1.) integra dos modelos. Uno, basado en la filosofía de Lonergan, está articulado en torno a los tres ejes de la conciencia, del conocimiento y de la comunicación. El otro modelo está basado sobre el enfoque metateórico del psicólogo social Kurt Lewin, quien afirma que la conducta humana (C) resulta (=) de la interacción $(\leftarrow \rightarrow)$ entre personas (P) y su ambiente (A) (Hunt, 1974). En nuestro modelo articulamos las seis variables $(\mathrm{P} \leftarrow \rightarrow \mathrm{A}=\mathrm{C})$ de Lewin con el enfoque de Lonergan, del modo siguiente: conciencia del sujeto que aprende $(\mathrm{P} \rightarrow)$, conocimiento del objeto $(=\mathrm{C})$ y el ambiente de comunicación $(\leftarrow A)$ entre sujeto-objeto.

Nuestra tesis es que los términos del modelo y las relaciones verticales y horizontales entre estos términos definen una ecología semántica, que tiene como objeto de estudio la comunicación en redes de diseño heurístico que entran en juego cuando los sujetos juntos buscan soluciones a problemas específicos (Morin, 2002). En el cuadro siguiente, se puede observar las relaciones verticales y horizontales de los términos que definen el modelo. Después explicaremos el significado de estos términos y más adelante revisaremos cómo el modelo está relacionado con los deseos, la afectividad, los valores y el desarrollo de significados culturales.

Tabla 1. Modelo ecológico-semántico de una ciencia y cultura transdisciplinaria

\begin{tabular}{|l|l|l|}
\hline $\begin{array}{l}\text { Conciencia intencional } \\
\text { (ciencia del sujeto) } \\
\text { autoapropiación }\end{array}$ & $\begin{array}{l}\text { Comunicación } \\
\text { (ciencia de objetivación) } \\
\text { método empírico generalizado }\end{array}$ & $\begin{array}{l}\text { Conocimiento de la } \\
\text { realidad } \\
\text { (ciencia del objeto) } \\
\text { universo del ser } \\
=\text { Resultado }\end{array}$ \\
\hline Conducta \\
\hline
\end{tabular}




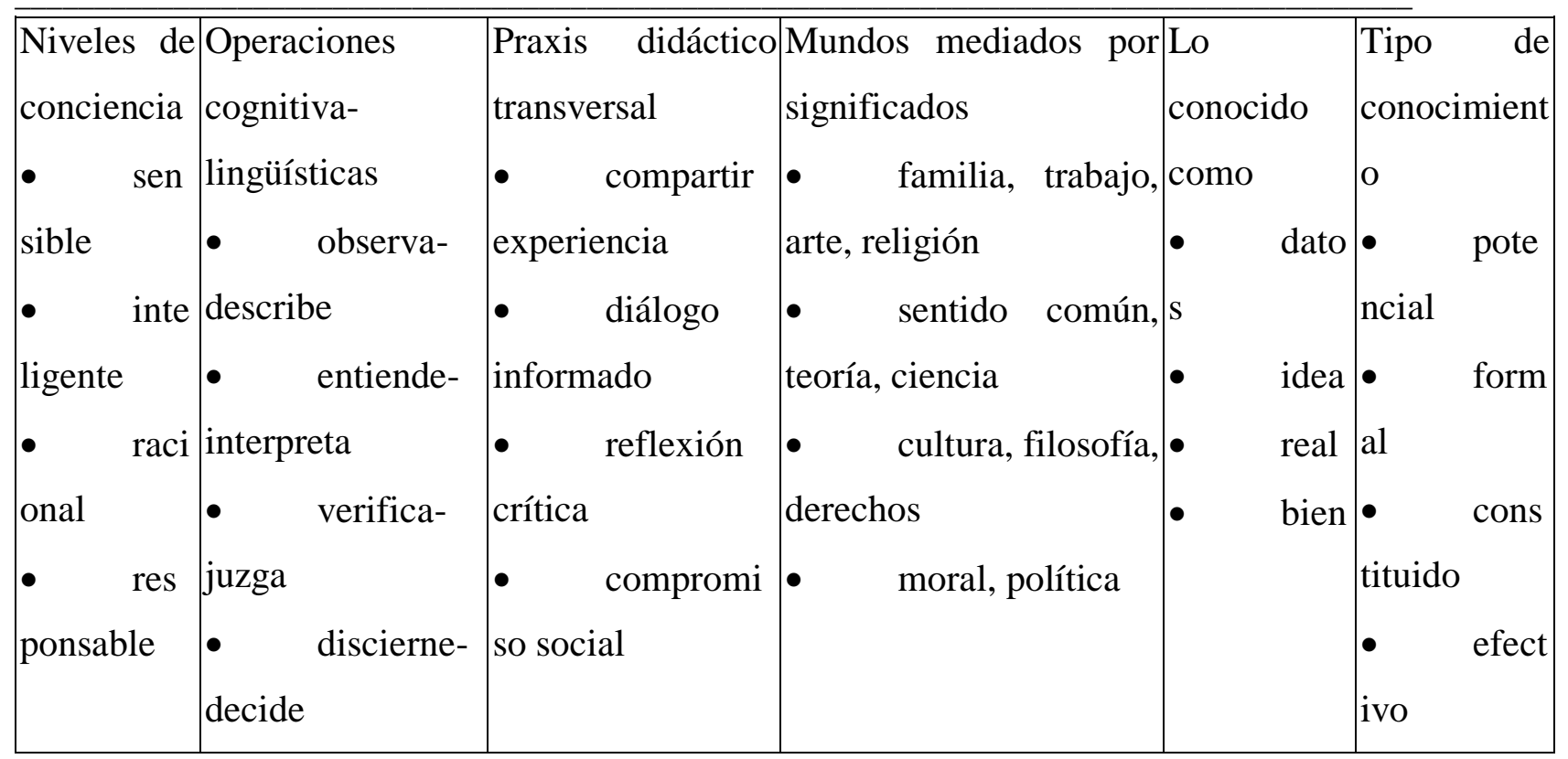

El modelo está estructurado en torno a la relación isomórfica entre los ejes de la conciencia del sujeto, del conocimiento del objeto y la comunicación entre ambos. Esta estructura permite explicitar una ecología semántica de un sistema integrado que es común a los distintos campos del conocimiento. Esta ecología está definida por las relaciones articuladas en torno a los niveles sensible, inteligible, racional y moral de la conciencia intencional. Así, en el modelo, las operaciones cognitivo-lingüísticas de observar-describir, entenderinterpretar, verificar-juzgar y discernir-decidir (ciencia del sujeto) relacionan las personas con la estructura de lo que conocen mediante datos e ideas y como realidad y bien (ciencia del objeto), y que la praxis comunicativa entre sujeto-objeto transcurre por medio de los diversos mundos de significación que habitan los sujetos (ciencia de objetivación).

Por el hecho de que esta estructura isomórfica es común a todas las formas de conocer, ofrece un marco que no impone presupuestos, conceptos y criterios ajenos, provenientes de otros campos del saber. Más bien, tiene como base un proceso común a todos los campos de saber y sobre el cual se puede fundamentar el desarrollo de una ciencia transdisciplinaria. Una ciencia que facilita una síntesis superior del conocimiento, por medio del diálogo, la reflexión crítica y el compromiso entre diversas formas de saber cuándo juntos buscan respuestas. 
El carácter heurístico del modelo nos remite a la curiosidad y a las preguntas que orientan el desarrollo del aprendizaje en todas las personas y en todos los contextos, pues estimula buscar respuestas a esa curiosidad, a esas preguntas, dudas y problemas. Las preguntas pueden surgir de la curiosidad de los niños, de las dudas del sentido común en la vida cotidiana; de los conflictos existenciales de la juventud, de los problemas económicos, políticos y culturales en contextos sociales específicos, o de la investigación sistemática de las ciencias, en las exigencias de los derechos y en el diseño de políticas públicas y, también en la búsqueda de la fe religiosa. Las redes semíticas comienzan a desarrollarse por medio de las operaciones cognitivo-lingüísticas que orientan la comunicación consciente en las formas prácticas, científicas, culturales, religiosas, políticas y filosóficas del conocer en contextos diversos. El diseño se refiere a las actividades guiadas por las preguntas, ideas, conceptos, teorías y criterios que surgen históricamente en estos contextos para responder al cuestionamiento heurístico que busca respuestas a los problemas del entorno.

Transcendencia en la integración cognitiva de deseos, afectividad y valores Una comprensión más plena de la filosofía de Lonergan requiere considerar tres dimensiones adicionales de su pensamiento en relación con el modelo anterior. La primera refiere al modo como los deseos y los sentimientos animan las operaciones cognitivo-lingüísticas de la conciencia intencional, conforme a una escala ascendente de valores. La segunda dimensión considera cómo los deseos, la afectividad, la cognición y el lenguaje influyen en el desarrollo de los horizontes diferenciados de significación cultural y cómo las desviaciones de este proceso inciden en la decadencia cultural. La tercera dimensión se relaciona con cómo la función dialéctica del método empírico generalizado afronta posiciones equivocadas, limitadas o sesgadas sobre el conocimiento, con el fin de promover cambios que favorezcan el desarrollo integral de la persona y su cultura. A continuación, examinaremos cómo estas dimensiones influyen sobre el desarrollo de nuestra capacidad humana de transcender en la integración del conocimiento.

A nivel de su conciencia sensible, los seres humanos sienten hambre, necesidades de protección y de descanso, impulsos sexuales y el deseo de saber. Las operaciones cognitivolingüísticas de este nivel constituyen un saber potencial y práctico basado en percepciones, 
$\overline{\text { sentimientos, observaciones, descripciones y reacciones a los estímulos y la información del }}$ entorno. A este nivel, en lo social los seres humanos para poder cubrir sus necesidades, trabajan colaborativamente en el desarrollo de tecnologías, en la gestión de sistemas económicos y para su defensa, lo que repercute en su vida cultural y entorno ecológico. Así, a lo largo de su historia, la humanidad ha desarrollado formas de vida nómade, de producción agrícola e industrial, y modos de comunicación corporal, oral, escrita, electrónica y digital. La conciencia intencional de este nivel social-sensible tiene un carácter pragmático y está organizado en actividades laborales y recreativas, y se expresa por medio del sentido común y por un lenguaje técnico y administrativo. También, a este nivel, se da la búsqueda de la belleza, con sus diversas formas de expresión artística: en la música, el baile, la escultura, la pintura, la poesía, la literatura, el teatro y el cine. Estas son expresiones vitales, inspiradas por sentimientos e imágenes, mediante las cuales los artistas invitan a tomar distancia de la vida práctica para imaginar otros mundos de significación posibles. De modo similar, emergen símbolos, mitos y rituales de inspiración religiosa que dan testimonios de fe sobre dimensiones misteriosas o reveladas que afirman como sagradas y transcendentes. Los valores vitales, correspondientes a este nivel, son pragmáticos y ambiguos, porque sus criterios son lo agradable y lo placentero, lo que satisface las necesidades humanas básicas. La experiencia de la transcendencia en este primer nivel es también básica y vital: en la atracción y el placer sexual, y en la procreación. Experiencias de transcendencia, por ejemplo: al salir de sí mismo cuando se atiende al objeto o sujeto que se desea conocer; en la producción laboral, en la expresión artística o en los ritos de celebración religiosa. En este horizonte empírico-sensorial se da la posibilidad de una integración vivencial y práctica.

A nivel de la conciencia inteligible, el sentimiento de asombro y la curiosidad despiertan la dinámica heurística de inquirir, buscar y encontrar respuestas frente a lo desconocido o a un problema por resolver. Las operaciones cognitivas de este nivel se caracterizan por la capacidad de interrogar, entender, concebir, explicar o interpretar mediante imágenes, símbolos e ideas. En cuanto a la intencionalidad, esta se manifiesta mediante preguntas, intuiciones y especulaciones, y en la formulación de explicaciones e interpretaciones mediadas por conceptos, hipótesis y teorías que comprenden patrones inteligibles en lo que se quiere comprender. Es un nivel de saber formal en el que se puede diferenciar el desarrollo 
histórico, polimórfico y especializado de la conciencia intencional: en el sentido común y la sabiduría popular, en visiones políticas del orden social, en interpretaciones estéticas, en fórmulas matemáticas, en explicaciones científicas de la naturaleza, en el desarrollo de los significados culturales, en el pensamiento filosófico y en la comprensión teológica de las experiencias de fe religiosa. Lo que se valora son las ideas, mediante las cuales se puede transcender en la comprensión de patrones inteligibles del conjunto. En este horizonte se da la posibilidad de una integración mayor, basada en una comprensión coherente, sistemática, teórica y científica del mundo como conjunto sensible e inteligible.

A nivel de la conciencia racional, la necesidad de superar dudas y el deseo de conocer la verdad incita la reflexión crítica, que busca verificar y juzgar con fundamentos lo que es probable, verdadero, real y de valor. Este tercer nivel de saber reflexivo afirma con evidencia y criterios los fundamentos del conocimiento, los significados, los valores y los derechos. Responde a las dudas y al cuestionamiento crítico sobre si las teorías son explicaciones adecuadas de la naturaleza y de la realidad, sobre si las ideas son interpretaciones válidas de los significados culturales y religiosos, y sobre si los valores declarados realmente promocionan el desarrollo integral de la persona y del bien común. A este nivel corresponde preguntar por la validez del método que estamos proponiendo para fundamentar un conocimiento objetivo basado en la conciencia intencional del sujeto humano. Todo cuestionamiento crítico que busca refutar o falsar el método empírico generalizado, necesariamente tienen que recurrir a las operaciones de observar la evidencia, interpretar esta con inteligencia, juzgar con criterios y discernir para decidir (Lonergan, 1988, p.26). Este cuestionamiento lleva necesariamente al sujeto crítico a confrontar su propia conciencia intencional, experiencia de conocer y opción de asumir o no esta actividad como medio que posibilita el autoconocimiento, la autoapropiación y la autotranscendencia en el acto de conocer. Es la base sobre la cual se puede validar, criticar, corregir y mejorar los criterios fundantes de las ciencias, de los significados culturales y religiosos, y de los valores morales y políticos. A este nivel se da la posibilidad de una integración sensible, inteligible y actualizada del conocimiento. 
A nivel de la conciencia moral, es el deseo de hacer lo que es verdaderamente bueno lo que orienta el desarrollo de la capacidad protagónica de los sujetos, para guiarse con criterios de juicio, al discernir, proponer, decidir, comprometer y actuar con libertad responsable. Es un nivel de saber existencial que está situado culturalmente, y mediante el cual la persona puede constituirse en fuente de valores, definir su carácter moral y constituirse como sujeto histórico, en tanto ejerza su libertad para promover el bien con los demás respecto a los desafíos de su entorno (Lonergan, 1988, pp.11-20). El desarrollo de su protagonismo moral depende del modo como discierna y decida actuar coherentemente, según unos criterios de juicio fundados en una comprensión inteligente y crítica de los hechos y la realidad. Su autorealización será obra de su conciencia moral en la medida en que transcienda en la promoción del bien. A este nivel se da la posibilidad de una integración sensible, inteligible, actual y efectiva, en cuando uno transciende auténticamente, cuando es atento, inteligible, racional y responsable en la toma de decisiones libres.

\section{Progreso, decadencia y cambio cultural}

Lo revisado aporta un marco que identifica procesos, conceptos y criterios que pueden orientar el desarrollo de una ciencia transdisciplinaria, promotora del desarrollo integral de la persona y su cultura. Ahora para ampliar nuestra mirada con este marco, consideraremos el pensamiento de Lonergan sobre dos puntos adicionales: el desarrollo diferenciado de los horizontes de significación y su análisis de aquello que incide sobre el progreso y la decadencia histórica de estos horizontes.

Lonergan distingue entre horizontes de significación mediados por el sentido común, el arte, el mito, la teoría y la interioridad. Cada horizonte con su comprensión propia y lenguaje específico, está constituido por la misma actividad cognitiva de observar, interpretar, juzgar y decidir. Distinguimos a continuación las características propias de cada horizonte. El sentido común comprende las cosas en relación pragmática con las necesidades de los sujetos y su grupo. Las expresiones vitales del arte exploran imaginativamente nuevas posibilidades más allá de los límites dados por los horizontes existentes del sentido común. Los mitos son interpretaciones simbólicas significativas sobre el origen, el sentido y el propósito de la vida. La teoría busca explicaciones sistemáticas de la naturaleza, de los significados humanos y 
de la realidad. La interioridad es la conciencia intencional apropiada que transciende cuando conoce auténticamente. Ella es la base constitutiva de todos los horizontes de conocimiento y de significación. Fundamenta la posibilidad de una mirada en que se sabe mover con apreciación crítica entre los diferentes horizontes de significación común, científica, cultural, política y religiosa (Lonergan, 1988, pp.61-76). Afirmamos que la interioridad apropiada aporta las bases del diálogo transdisciplinario que facilita la comprensión y la colaboración entre los diversos horizontes de significación. De acuerdo con la filosofía de Lonergan, el diálogo tendrá un componente dialéctico que promueve cambios personales y culturales que buscan superar la decadencia y promover el progreso humano.

Dentro de este marco, el progreso está fundado sobre sujetos auténticos que transcienden al realizar la potencia activa de su ser. Transcienden cuando viven coherentemente de acuerdo con las exigencias de su conciencia intencional que requiere que uno sea atento, inteligente, razonable y responsable. El progreso depende de un fluir continuo y consolidado en la realización de estos preceptos frente las situaciones de la vida y los desafíos históricos del entorno. La decadencia se origina en las desviaciones de la conciencia intencional: en la desatención, la insensibilidad, la incomprensión, la racionalización, la irresponsabilidad y el resentimiento (Lonergan, 1988, pp.21-26).

Algunos síntomas de decadencia incluyen las formas más básicas de alienación, que tienen su origen: 1) en la negligencia y desviaciones de la conciencia intencional; 2) en el sentido común, cuando se opone a las exigencias sistemáticas y críticas, por creerse irrefutable en el mundo práctico y político; 3) en el egoísmo individual y grupal, cuando de forma unilateral atenta contra el bien común y racionaliza su proceder; 4) en la ley, cuando se convierte en instrumento de una clase en vez de un medio para asegurar la justicia; 5) en la ideología, cuando justifica este comportamiento como mal necesario para un bien mayor; 6) en el poder corrupto, que busca el provecho material, controla a los medios de comunicación, fundamenta el orden político, inspira la cultura de moda y las filosofías dominantes, desvía a la fe religiosa y orienta los procesos educativos (Lonergan, 1988, pp.57-60). 
En relación con esta comprensión del progreso y de la decadencia, el cambio puede darse a nivel psíquico, cognitivo, moral o religioso y de forma combinada. A nivel psíquico un cambio en los sentimientos puede tener repercusiones sobre las actitudes, operaciones cognitivas y escala de valores fundamentales de una persona y su cultura. A nivel cognitivo el realismo crítico supera los mitos que reducen el conocimiento al dato empírico de los sentidos o a los constructos ideales de la inteligencia. Afirma que el conocimiento es un proceso de autotranscendencia que pasa por la actividad compuesta de experimentar, entender y juzgar. A nivel moral, se puede dar un cambio desde una conducta que solo busca la satisfacción de los placeres, las necesidades y los intereses propios, a un actuar orientado por criterios de decisión informados, razonables, libres y responsables, mediante los cuales se busca lo que es verdaderamente bueno para todos. La conversión religiosa, en la tradición judeo-cristiana, está fundada en la experiencia de reconocerse amado por Dios. Implica un aprendizaje permanente del modo como el amor lleva a la autotranscendencia y la autorrealización; y esto se logra en la medida en que el amor integra y transforma el sentir, el pensar, el decidir y el actuar. De esta forma una religión auténtica puede desempeñar una función sanadora para reparar el daño causado por la decadencia al promover el desarrollo integral de la persona y su cultura (Lonergan, 2006, 231-238).

Una persona "realiza su autenticidad en la auto-transcendencia" y que "esta capacidad llega a ser actualidad cuando uno se enamora (Lonergan, 1988, pp.105-107).” Así el desarrollo pleno de la persona se da cuando las operaciones de la conciencia intencional están animadas por el amor. El amor puede animar y orientar el desarrollo y la integración de los cuatro niveles de la conciencia intencional: cuando se observa atentamente la vida con amor; cuando se busca con amor una comprensión inteligible del mundo; cuando se fundamenta los criterios de juicio con amor y cuando se discierne y decide cómo amar con libertad responsable. Como estado dinámico, el amor puede animar el diálogo y la colaboración en múltiples dimensiones: en la relación de la pareja y entre padres e hijos; en el compromiso de los ciudadanos y los gobernantes con el desarrollo de su nación; en la vocación laboral de trabajadores, profesionales, artistas y científicos; y en relación con Dios con la entrega de sí mismo en el servicio de los demás (Lonergan, 1988, pp.106 y 289). Cuando el amor anima estas diversas formas de ser, el criterio de juicio evaluativo es el mismo que para los niveles 
del conocimiento sensible, inteligible, racional y moral: nace de una subjetividad auténtica que conoce en la medida que transciende en relación con lo que desea conocer, amar y entregarse (Lonergan, 1988, pp.42-43).

\section{Hacia una ciencia y cultura transdisciplinaria}

A lo largo de este estudio hemos descubierto que la transdisciplinariedad implica un cambio de paradigma científico y cultural en la producción de conocimientos transformativos. Es un enfoque que busca superar la segmentación del saber en departamentos, al promover el desarrollo y la integración del conocimiento. Por medio del diálogo, convoca a diversos actores del mundo científico, cultural y político para colaborar frente los desafíos complejos de nuestros tiempos. Desde las primeras aproximaciones, por definir las características y el propósito de la transdisciplinariedad, se ha registrado grandes avances en el desarrollo de proyectos, investigaciones y publicaciones. Nuestra revisión de esta historia ha identifica unos criterios a considerar en el diseño, la realización y la evaluación de proyectos transdisciplinarios que responden a problemas específicos.

Nuestro estudio constata que no se puede fundamentar o reducir la transdisciplinariedad a una ciencia particular, a una teoría especulativa o a propuestas pragmáticas. Frente el desafío epistemológico de fundamentar la transdisciplinariedad, proponemos un método que promueve apropiar la estructura dinámica de la propia conciencia intencional, que es común a todos los campos del saber humano. Así, frente a los problemas complejos, el foco para la integración del conocimiento es el protagonismo de sujetos sensibles, inteligentes, racionales y responsables, quienes conocen en la medida que transcienden auténticamente en relación con lo que desean conocer.

A partir de este método esbozaremos un modelo que distingue niveles sensible, inteligible, racional y moral en la comunicación consciente del conocimiento. Este modelo tiene como finalidad orientar el diálogo y la colaboración entre diferentes formas de saber, en que hemos diferenciado el sentido común, competencias técnico-profesionales, inspiraciones artísticas, creencias religiosas, derechos políticos, teorías científicas, significados culturales y el razonamiento filosófico. En un proyecto transdisciplinario el propósito del diálogo será 
promover una apreciación crítica de los méritos y las limitaciones de cada forma de saber y lo que contribuye al desarrollo de conocimientos transformativos.

Un proyecto transdisciplinario que promueve el diálogo y la colaboración para el desarrollo e integración de conocimientos transformativos, en relación con un problema complejo debe tener apertura a todas las formas de conocer. Es capaz de escuchar diversos actores y sus diferentes formas de saber para discernir en sus observaciones, interpretaciones, juicios y propuestas lo que contribuyen sobre un problema determinado. Por ejemplo, los desafíos que presenta el cuidado del medio ambiente no pueden resolverse solo por el conocimiento científico. También requiere la colaboración no científica: de gobiernos que deciden sobre el diseño y la implementación de políticas públicas; de la creatividad artística que despierta la capacidad de imaginar las posibilidades de un mundo distinto; del cambio de actitudes en el sentido común que lleva a nuevas conductas; y de las creencias religiosas que inspiran convicciones y compromisos. Una ciencia y cultura transdisciplinaria que pretende aportar un marco para el diálogo y la colaboración, no puede ignorar ninguna de estas voces. Reconoce por una parte que todas las formas del conocimiento tienen su origen en observaciones, interpretaciones, juicios y decisiones. Por otra parte, valora los aportes y al mismo tiempo cuestiona críticamente los sesgos que limitan las diversas formas de conocer, sean éstas de sentido común, o religiosas, o políticas, o científicas, o filosóficas.

Este enfoque transdisciplinario ofrece un marco para superar las limitaciones e integrar los aciertos de la educación humanista y de la teoría crítica que representan los componentes personales y sociales de una sociedad democrática. La educación humanista promueve la autorrealización de toda la persona, pero tiende a carecer de un análisis sociopolítico y asume que el cambio individual lleva a una transformación institucional. La teoría crítica proporciona un análisis estructural de cómo la educación reproduce desigualdades sociales, pero no considera estrategias pedagógicas para promover el cambio en el nivel de la experiencia vivida. ${ }^{8}$ El método empírico generalizado promueve la integración de ambas dimensiones. Por una parte, facilita el desarrollo personal en la medida que los educandos

8 Lee BELL and Nancy SCHNIEDEWIND, "Realizing the Promise of Humanistic Education: A Reconstructed Pedagogy for Personal and Social Change," (Journal of Humanistic Psychology 29, 1989), 200-223. 
ejercen sus capacidades de observar atentamente, preguntar para entender e interpretar con inteligencia y juzgar con criterios que orienten el desarrollo de sus compromisos. Esto facilita la comprensión y apropiación de su propia intencionalidad consciente y el desarrollo de la misma en la promoción de la justicia social y el cambio sistémico en relación con los problemas específicos de su entorno. De esta forma pueden desarrollar su conciencia intencional y transcender como ciudadanos sensibles, inteligentes, críticos y comprometidos.

Referencias bibliográficas

Asumah, Seth \& Nagel, Mechthild (Eds.) (2015). Diversity, Social Justice, and Inclusive Excellence: Transdisciplinary and Global Perspective. Albany. State University of New York.

Burger, Paul, and Rainer Kamber (2003) Cognitive Integration in Transdisciplinary Science. Issues in Integrative Studies. Vol. 21. Pp. 43-73. Recuperado de https://pdfs.semanticscholar.org/2eef/d5ebdccacf5327fc0c423d9ad0dee5cdf338.pdf.

Bergmann, Matthias. Jahn, Thomas. Knobloch, Tobias. Krohn, Wolfgang. Pohl, Christian. Schramm, Engelbert (2012). Methods for Transdisciplinary Research. A Primer for Practice. Frankfurt. Campus Verlag. Recuperado de http://www.mrtc.mdh.se/ gdc01/work/Methods\%20for\%20Transdisciplinary\%20Research. pdf.

Bernstein, Jay Hillel (2015). Transdisciplinarity: A Review of Its Origins, Development, and Current Issues. Journal of Research Practice. Vol. 11, $\mathrm{N}^{\circ} 1$. Recuperado de http://jrp.icaap.org/index.php/jrp/article/view/510/412.

Brown, Valerie. Harris, John. Russell, Jacqueline (Eds.) (2011). Tackling Wicked Problems through the Transdisciplinary Imagination. Journal of Natural Resources Policy Research. Vol. $3 . \quad \mathrm{N}^{\circ} \quad 10 . \quad$ Recuperado de https://www.researchgate.net/profile/Katharine_Farrell/publication/238046777_Tackling Wicked_Problems_Through_the_Transdisciplinary_Imagination/links/54de1e820cf22a267 21ecc26/Tackling-Wicked-Problems-Through-the-Transdisciplinary-Imagination.pdf.

Camus, Michel y Nicolescu, Basarab (Eds.). (1997). Declaración y recomendaciones del Congreso Internacional ¿Qué Universidad para el mañana? Hacia una evolución transdisciplinar de la Universidad. Arrábida, Portugal. Congrès de Locarno. Recuperado de http://ciret-transdisciplinarity.org/locarno/loca7sp.php. 
Carrizo, Luis. (2003). Pensamiento complejo y transdisciplinariedad. Universidad de Buenos Aires. Argentina. Recuperado de http://ecosad.org/phocadownloadpap/otrospublicaciones/carrizo-pensamientocomplejoytransdisciplinariedad.pdf.

Carrizo, Luis; Espina Prieto, Mayra \& Klein, Julie T. (2004). Gestión de las Transformaciones Sociales. UNESCO. Recuperado de http://unesdoc.unesco.org/images/0013/001363/136367s.pdf.

Conrad, P.A. Mazet J.A, Clifford D, Scott C, Wilkes M. (2009). Evolution of a transdisciplinary 'One Medicine-One Health' approach to global health education at the University of California. Preventive Veterinary Medicine. Vol. 92, N ${ }^{\circ}$ 4. Pp. 268-274. Recuperado de https://www.ncbi.nlm.nih.gov/pubmed/19819575.

Deane-Drummond, Celia. Bergmann, Sigurd. Szerszynski, Bronislaw. (Eds.). (2015). Technofutures, Nature and the Sacred: Transdisciplinary Perspectives. Surrey, UK. Ashgate Publishing. Recuperado de http://www.tandfonline.com/doi/full/10.1080/13617672.2015.1091116?scroll=top\&needAc cess $=$ true.

Gibbons, Michael et al. (1994). The new production of knowledge: The dynamics of science and research in contemporary societies. London. Sage.

Godemann, Jasmin (2008). Knowledge integration: a key challenge for transdisciplinary cooperation. En Environmental Education Research. Vol. 14, №. 6. Pp. 625-641. Recuperado de http://www.tandfonline.com/doi/full/10.1080/13504620802469188.

Judge, Anthony (2005). Transdisciplinarity-3 as the emergence of patterned experience. En Journal of the Interdisciplinary Crossroads. Vol 2, No. 2. Recuperado de https://www.laetusinpraesens.org/docs/tranpat1.php.

Judge, Anthony (2010). Enacting Transformative Integral Thinking through Playful Elegance. Kairos. Recuperado de http://kairos.laetusinpraesens.org/enplay_1_h_1.

Hinkel, Jochen (2008). Transdisciplinary Knowledge Integration. Cases from Integrated Assessment and Vulnerability Assessment. Netherlands. Wageningen University. Recuperado de http://ciret-transdisciplinarity.org/biblio/biblio_pdf/Hinkel.pdf.

Hirsch Hadorn, Gertrude (2002). Unity of Knowledge in Transdisciplinary Research for Sustainability. En: Encyclopedia of Life Support Systems, Oxford UK. Recuperado de https://www.eolss.net/Sample-Chapters/C04/E6-49.pdf

Hirsch Hadorn, Gertrude \& Biber-Klemm, Susette \& Grossenbacher-Mansuy, Walter \& Hoffmann-Riem, Holger \& Joye, Dominique \& Pohl, Christian \& Wiesmann, Urs \& Zemp, Elisabeth. (2008). The Emergence of Transdisciplinarity as a Form of Research. En: Hirsch

Hadorn G. et al. (Eds). Handbook of Transdisciplinary Research (pp. 19-39). Dordrecht, Holanda. $\quad$ Springer. Pp. 19-39. Recuperado de 
https://www.researchgate.net/profile/Holger_Hoffmann-

Riem/publication/225880759_The_Emergence_of_Transdisciplinarity_as_a_Form_of_Res earch/links/0deec5275f9a2da592000000/The-Emergence-of-Transdisciplinarity-as-a-Formof-Research.pdf.

Hunt, David \& Sullivan, Edmund (1974). Between Psychology and Education. Toronto. University of Toronto Press. Pp. 19 -

Huutoniemi, Katri \& Tapio, Petri. (Eds.). (2014). Transdisciplinary Sustainability Studies: A Heuristic Approach. New York. Routledge.

Jantsch, Erich (1972). Towards Interdisciplinarity and Transdisciplinarity in Education and Innovation. En L. Apostel, G. Berger, A. Briggs, y G. Michaud (Eds.). Interdisciplinarity. Problems of Teaching in Universities. Organization for Economic Co-operation and Development. Centre for Educational Research and Innovation. Pp. 97-121. Ver la traducción español de Francisco J. González. Recuperado de http://publicaciones.anuies.mx/acervo/revsup/res034/txt7.htm.

Kastenhofer, K. (2009). Science Policy for Transdisciplinary Research. En Unity of Knowledge. En Transdisciplinary Research for Sustainability. Vol. II. Encyclopedia of Life Support Systems. Recuperado de https://www.researchgate.net/profile/Karen_Kastenhofer/publication/265534970_SCIENC E_POLICY_FOR_TRANSDISCIPLINARY_RESEARCH/links/54b29b500cf220c63cd268 51/SCIENCE-POLICY-FOR-TRANSDISCIPLINARY-RESEARCH.pdf

Kirst, Maritt \& Schaefer-McDaniel, Nicole \& Hwang, Stephen \& O’Campo, Patricia (Eds.). (2011) Converging Disciplines: A Transdisciplinary Research Approach to Urban Health Problems. Centre for Research on Inner City Health, Toronto. New York. Springer. Recuperado de http://dlib.bpums.ac.ir/multiMediaFile/20772388-4-1.pdf.

Klein, Julie Thompson (1998). Notes Toward a Social Epistemology of Transdisciplinarity. Rencontres Transdisciplinaires, Bulletin $N^{\circ} 12$. Centre International de Recherches et Études transdisciplinaires. Recuperado de http://ciret-transdisciplinarity.org/bulletin/b12c2.php.

Klein, Julie Thompson (2014). Discourses of transdisciplinarity: Looking Back to the Future. En Futures. $\quad \mathrm{N}^{\circ}$ 63. Pp. 68-74. Recuperado de http://www.graniczne.amu.edu.pl/PPGWiki/attach/Transdyscyplinarno\%C5\%9B\%C4\%87/ Discourses\%20of\%20transdisciplinarity\%20Looking\%20Back\%20to\%20the\%20Future.pd $\underline{\mathrm{f}}$

Krott, M. (2003). Evaluation of transdisciplinary research. En: Encyclopedia of life support systems. Oxford: EOLSS. Recuperado de http://www.eolss.net/Sample-Chapters/C04/E649-02-07.pdf.

Kueffer, Christoph \& Hirsch Hadorn, Gertrude \& Bammer, Gabriele \& van Kerkhoff, Lorrae \& Pohl, Christian. (2007). Towards a Publication Culture in Transdisciplinary Research. En 


\begin{tabular}{llllllll}
\hline GAIA. & Vol. & 16. & $\mathrm{~N}^{\circ}$ & 1. & Pp. & $22-26$. & Recuperado
\end{tabular}
https://www.ethz.ch/content/dam/ethz/specialinterest/usys/tdlab/docs/pub/2007_Gaia_16.pdf.

Lawrence, Roderick (2010). Deciphering Interdisciplinary and Transdisciplinary Contributions. Transdisciplinary Journal of Engineering \& Science. Vol. 1, No. 1. Pp. 125130. Recuperado de https://www.ed.ac.uk/files/imports/fileManager/RJL-2010InterTrans.pdf.

Lichnerowicz, Andre (1972). Mathematic and transdisciplinarity. En L. Apostel, G. Berger, A. Briggs, y G. Michaud (Eds.). Interdisciplinarity. Problems of Teaching in Universities. Organization for Economic Co-operation and Development. Centre for Educational Research and Innovation. Pp. 121-127.

Lonergan, Bernard (1992). Insight: A Study of Human Understanding. Frederick E. Crowe and Robert M. Doran. (Eds.). Vol. 3. Frederick E. Crowe and Robert M. Doran. (Eds.) University of Toronto Press.

(1993). Topics in Education. The Cincinnati Lectures of 1959 on the Philosophy of Education. Collected Works. Vol. 10. Robert Doran y Frederick Crowe. (Eds.). University of Toronto Press.

(1999). Insight: Estudio sobre la comprensión humana. Traducción de Francisco Quijano. Salamanca, Universidad Iberoamericana-Sígueme.

Salamanca. Sígueme.

(2006). Método en Teología. Traducción de Gerardo Remolina.

Mahan, Jack Lee (1970). Toward transdisciplinary inquiry in the humane sciences. Doctoral dissertation, United States International University.

Maturana, Humberto (1997). El sentido de lo humano. Santiago. Dolmen.

Mauser, Wolfram \& Klepper, Gernot \& Rice, Martin \& Schmalzbauer, Bettina Susanne \& Hackmann, Heide \& Leemans, Rik \& Moore, Howard (2003) Transdisciplinary global change research: the co-creation of knowledge for sustainability. En Current Opinion in Environmental Sustainability. Vol. 5. № 3-4. Pp. 420-431. Recuperado de https://www.ifwmembers.ifw-kiel.de/publications/transdisciplinary-global-change-research-the-co-creationof-knowledge-for-sustainability/1-s2.0-S1877343513000808-main.pdf.

Max-Neef, Manfred (2005). Foundations of transdisciplinarity. En Ecological Economics. Vol. 53. Pp. 5-16. 5 Recuperado de http://aoatools.aua.gr/pilotec/files/bibliography/transdiscipl_MaxNeef_ecolEcon3345059585/transdiscipl_MaxNeef_ecolEcon.pdf 
McMichael, Anthony (1998). What makes Transdisciplinarity succeed or fail? En Transdisciplinarity. Stimulating Synergies, Integrating Knowledge. UNESCO. Recuperado de http://unesdoc.unesco.org/images/0011/001146/114694eo.pdf.

Morin, Edgar (2001). Introducción al pensamiento complejo. Barcelona: Gedisa.

Morin, Jim. Icaza, Bernardita. Marfán, Jukia. (1996). Conversemos de sexualidad. Programa para padres, profesores y estudiantes. Santiago. Centro de Investigación y Desarrollo de la Educación.

Morin, Jim (2002). Conscious intentionality as foundation for a trans-disciplinary science. En Going Beyond Essentialism: Bernard J. F. Lonergan, An Atypical Neo-Scholastic. Cloe. (Ed.) Naples. Taddei-Ferretti. Istituto Italiano per Gli Studi Filosofici. Pp. 331-338.

National Research Council (2014). Convergence: Facilitating Transdisciplinary Integration of Life Sciences, Physical Sciences, Engineering, and Beyond. Washington, DC. The National Academies Press. Recuperado de http://cirettransdisciplinarity.org/quoideneuf/NSF_Report_on_TD.pdf.

Newell, B. et al. (2005) A conceptual template for integrative human-environment research. En Global Environmental Change. Vol. 15, No 4. Pp. 299-307. Recuperado de http://citeseerx.ist.psu.edu/viewdoc/download?doi=10.1.1.456.8917\&rep=rep1\&type=pdf.

Newella, Barry \& Crumleyb, Carole L. \& Hassanc, Nordin \& Lambind, Eric F. \& PahlWostle, Claudia \& Underdalf, Arild \& Wasson, Robert. (2016). Increasing the usability of climate science in political decision-making. En Elementa: Science of the Anthropocene. Vol. 4. Recuperado de https://www.elementascience.org/articles/10.12952/journal.elementa.000127.

Nicolescu, Basarab (2009). The Relationship between Complex Thinking and Transdisciplinarity. Presentado en el Symposium on Complex Systems Modeling and Complexity Thinking. Paris. Fondation Maison des Sciences de 1'Homme, Maison Suger. Recuperado de http://cirettransdisciplinarity.org/ARTICLES/Nicolescu_fichiers/MSH15062009.pdf.

(2010). Methodology of Transdisciplinarity-Levels of Reality, Logic of the Included Middle and Complexity. Transdisciplinary Journal of Engineering \& Science. Vol. 1, No.1. Pp.19-38. Recuperado de http://www.basarabnicolescu.fr/Docs_Notice/TJESNo_1_12_2010.pdf.

Piaget, Jean (1972). The epistemology of interdisciplinary relationships. En L. Apostel, G. Berger, A. Briggs, y G. Michaud (Eds.). Interdisciplinarity. Problems of Teaching in Universities. Organization for Economic Co-operation and Development. Centre for Educational Research and Innovation. Pp. 127-139. 
Rodriguez, Darío \& Torres, Javier. (2003). Autopoiesis, la unidad de una diferencia: Luhmann y Maturana. En Sociologias. $\mathrm{N}^{\circ}$ 9, pp.106-140. Recuperado de http://www.scielo.br/scielo.php?pid=S1517-

$\underline{45222003000100005 \& \text { script }=\text { sci_abstract\&tlng=e. }}$.

Scholz, R. et al. (2006) Transdisciplinary case studies as a means of sustainability learning. International Journal of Sustainability in Higher Education, Vol 7, $\mathrm{N}^{\circ}$ 3. Pp. 226-251. Recuperado de http://cea.uprrp.edu/wp-content/uploads/2016/10/trans-case-studylearning.pdf.

Siew, Tuck-Fatt \& Döll, Petra (2012). Transdisciplinary research for supporting the integration of ecosystem services into land and water management in the Tarim River Basin, Xinjiang, China. Journal of Arid Land. Vol. 4, $\mathrm{N}^{\circ}$ 2. Pp. 196-210. Recuperado de https://www.researchgate.net/profile/Tuck_Fatt_Siew/publication/262699034_Transdiscipli nary_research_for_supporting_the_integration_of_ecosystem_services_into_land_and_wat er_management_in_the_Tarim_River_Basin_Xinjiang_China/links/5730516d08ae3736095 cc0bd/Transdisciplinary-research-for-supporting-the-integration-of-ecosystem-servicesinto-land-and-water-management-in-the-Tarim-River-Basin-Xinjiang-China.pdf.

Spash, C.L. (2012). New foundations for ecological economics. En Ecological Economics. Vol. $77 . \quad$ Pp. $36-47 . \quad$ Recuperado de http://epub.wu.ac.at/3711/1/Spash_EE_New_Foundations.pdf.

UNESCO (1998). Transdisciplinarity. Stimulating Synergies, Integrating Knowledge. Division of Philosophy and Ethics. Recuperado de http://unesdoc.unesco.org/images/0011/001146/114694eo.pdf.

Wiesmann, Urs \& Biber-Klemm, Susette \& Grossenbacher-Mansuy, Walter \& Hirsch Hadorn, Gertrude \& Hoffmann-Riem, Holger \& Joye, Dominique \& Pohl, Christian \& Zemp, Elisabeth (2008). Enhancing Transdisciplinary Research: A Synthesis in Fifteen Propositions. En Handbook of Transdisciplinary Research. pp 433-441. Recuperado de https://www.researchgate.net/profile/Holger_Hoffmann-

Riem/publication/225880759_The_Emergence_of_Transdisciplinarity_as_a_Form_of_Res earch/links/0deec5275f9a2da592000000/The-Emergence-of-Transdisciplinarity-as-a-Formof-Research.pdf. 